\title{
Energy deposition characteristics of nanosecond dielectric barrier discharge plasma actuators: Influence of dielectric material
}

\author{
G. Correale, ${ }^{a)}$ R. Winkel, ${ }^{\text {b) }}$ and M. Kotsonis ${ }^{\text {c) }}$ \\ Department of Aerospace Engineering, Delft University of Technology, Kluyverweg 1, 2629 HS Delft, \\ The Netherlands
}

(Received 10 March 2015; accepted 11 August 2015; published online 24 August 2015)

\begin{abstract}
An experimental study aimed at the characterization of energy deposition of nanosecond Dielectric Barrier Discharge (ns-DBD) plasma actuators was carried out. Special attention was given on the effect of the thickness and material used for dielectric barrier. The selected materials for this study were polyimide film (Kapton), polyamide based nylon (PA2200), and silicone rubber. Schlieren measurements were carried out in quiescent air conditions in order to observe density gradients induced by energy deposited. Size of heated area was used to qualify the energy deposition coupled with electrical power measurements performed using the backcurrent shunt technique. Additionally, light intensity measurements showed a different nature of discharge based upon the material used for barrier, for a fixed thickness and frequency of discharge. Finally, a characterisation study was performed for the three tested materials. Dielectric constant, volume resistivity, and thermal conductivity were measured. Strong trends between the control parameters and the energy deposited into the fluid during the discharge were observed. Results indicate that efficiency of energy deposition mechanism relative to the thickness of the barrier strongly depends upon the material used for the dielectric barrier itself. In general, a high dielectric strength and a low volumetric resistivity are preferred for a barrier, together with a high heat capacitance and a low thermal conductivity coefficient in order to maximize the efficiency of the thermal energy deposition induced by an ns-DBD plasma actuator. (C) 2015 AIP Publishing LLC. [http://dx.doi.org/10.1063/1.4929362]
\end{abstract}

\section{INTRODUCTION}

The number of studies meant to characterise plasma actuators has grown tremendously during the past years. Interest in these devices comes from their flow control capabilities demonstrated both experimentally and numerically. ${ }^{1,2}$ Two main kinds on plasma actuators are commonly investigated: Alternating Current Dielectric Barrier Discharge (AC-DBD) and nanosecond Dielectric Barrier Discharge (ns-DBD). The former is driven by alternating current and its actuation mechanism relies on production of a body force. . $^{3,4}$ The latter is powered by nanosecond pulses and was shown to have considerable flow control authority in the case of laminar leading edge separation. ${ }^{5-7}$ Its working principle relies on a thermal effect. ${ }^{8}$ Nevertheless, physical actuation mechanism is still subject of investigations.

During early work on ns-DBD, ${ }^{6-8,19}$ the physical flow actuation mechanism of ns-DBDs was attributed to the fast pressure gradient following the energy deposition due to the discharge (i.e., the induced shockwave). However, recent investigations gave numerical and experimental evidences that the flow control authority shown by ns-DBD plasma actuator does not purely rely on the formation of the shockwave. Rather, the effect can be due to a combination of pressure, viscosity, and density gradients produced by the residual heat deposited within the discharge volume by the

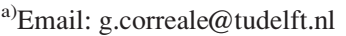

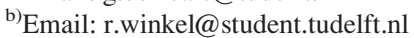

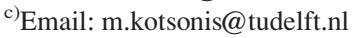

discharge $^{9,10,14}$ (same conclusion was reported ${ }^{13}$ and later acknowledged $^{29}$ by others).

In recent work by the authors, ${ }^{9,10}$ evidence was given of the existence of a strong thermal effect due to nanosecond plasma actuation using Schlieren imaging. The discharge takes place in nanosecond time-scale resulting in rapid energy deposition within a localised volume of air. The temperature rising time is in the order of microseconds, while gas expansion is in the order of milliseconds. Due to these timescales, the heated gas does not have enough time to expand resulting in a strong pressure rise within the discharge volume that generates a weak compression wave. ${ }^{8}$ Such wave serves as the footprint of an ns-DBD actuation and was subject of several studies in recent works. ${ }^{7,8,11,12}$ Using pulse widths of several microseconds, Benard et al. ${ }^{12}$ were able to demonstrate that, in fact, the compression wave is a superposition of two individual waves emanating from both the rising and falling portions of the high voltage pulse. Zheng et $a l .{ }^{13}$ have reproduced numerically the experiments of Benard et al. ${ }^{12}$ investigating parameters, such as voltage amplitude, rising time, and pulse length of a shockwave. They concluded that a disturbance induced by those compression waves was unable to justify the control authority shown by ns-DBD, as verified by Popov and Hulshoff. ${ }^{14}$ Correale et al. ${ }^{10}$ gave experimental evidence of the formation of a strong thermalized area in the vicinity of the actuator after each pulse discharged. They applied a plasma actuator in a stable laminar boundary layer and observed a flow perturbation originating from the thermal input. They 
argued that a possible flow control mechanism of ns-DBD actuators is density/viscosity gradients triggering, several milliseconds after the discharge, natural hydrodynamic instabilities of the flow under control.

Thus, flow actuation mechanism is based on a thermal disturbance. Such thermal disturbance is a function of energy deposition which itself is a function of several parameters of the ns-DBD plasma actuator. For instance, dielectric material and thickness of barrier are of particular importance.

In the field of AC-DBD actuators, several studies recognised the importance of the dielectric barrier material in order to optimise the actuator's efficiency and strength. ${ }^{15,16}$ A major outcome of these studies was the conclusion that dielectric barriers with high dielectric strength, low dielectric constant, and increased thickness (i.e., Plexiglass, Teflon, and Nylon) produce a larger body force than conventional Kapton-based actuators. Opaits et al. ${ }^{17}$ recognised the accumulation of charge on a dielectric barrier as a major source of inefficiency for AC-DBD plasma actuators. In order to overcome this limitation, they proposed the use of a slightly conductive silicon coating as to allow the bleed-off of charges accumulated on the surface of a dielectric barrier. Results showed that the new configuration of the plasma actuator suppresses charge build-up and that it can efficiently sustain a DC voltage discharge.

On the same line, Starikovskiy and Miles ${ }^{18}$ proposed, for both AC and nanosecond variant of plasma actuators, the use of a dielectric barrier constructed using an active composite material. In this way, they demonstrated the possibility to suppress the self-limiting characteristic of an AC-DBD, due to charge accumulation on the dielectric barrier. They combined $\mathrm{SiO}_{2}$ with dielectric material using ionimplants and ohmic connections in order to build a dielectric multilayer barrier allowing a partial depletion of the accumulated charge. They selected $\mathrm{SiO}_{2}$ due to its capability to resist to high voltages, fast switching times, high thermal conductivity, and robustness. With such complex dielectric barrier, they demonstrated the possibility to enhance the performance of plasma actuators manipulating the electrical proprieties of the barrier itself. However, their results were not expanded to the ns-DBD variant of plasma actuator.

In contrast to AC-DBD actuators, no systematic study on the effect of dielectric material for ns-DBD actuators is available to date. Yet, the qualitative characterization of energy deposition as function of the properties and the geometry of the material used for the barrier are of primary importance towards the efficiency optimization of this technology, and it is the objective of this work. Towards this goal, three different materials were selected and investigated in this study. These were the typically used polyimide film (Kapton), PA2200, and silicone rubber.

Schlieren measurements were carried out in a closed still-air box in order to qualify trends of energy deposition as function of dielectric material and thickness. Back-current shunt technique was employed in order to measure the energy input per pulse (composed by the energy deposited within the discharge volume and the energy dissipated within the actuator itself). The morphological features of the discharge were further studied using direct imaging. Finally, the used materials were tested in order to experimentally measure their thermal and electrical properties. Energy deposition shows a strong dependency on the thickness of dielectric barrier. Moreover, trends of energy deposition are strongly affected by the type of material used for the dielectric barrier. The calculated input power gives an understanding of net energy performance of an ns-DBD relatively to dielectric barriers of different materials. Moreover, light intensity acquisitions suggest a different nature of discharge for different materials.

\section{EXPERIMENTAL SETUP}

This section provides an overview of the experimental facility, nanosecond plasma actuator geometry, layout, and details of measurement techniques.

\section{Test facility}

Experiments are carried out in a still-air box designed for the purpose of testing DBD plasma actuators. It is made of clear Plexiglas so to allow optical access from every side. Moreover, its size is $500 \times 500 \times 1000 \mathrm{~mm}^{3}$ and it is fixed on a self-levelling optical table. Optics for PIV and Schlieren measurements are permanently arranged on the table. The set-up is set within the Flow Control Actuator Testing (FloCAT) facility of Delft University of Technology.

\section{Nanosecond plasma actuator}

A ns-DBD plasma actuator is constructed using two electrodes made out of adhesive copper tape, separated by a dielectric barrier (Figure 1). The materials used for the barrier were Kapton polyimide, silicone rubber, and polyamide 12-based nylon PA2200. The latter is a material typically used in powder form in laser sintering applications and 3D printing (EOS-PA2200 Spec. Sheet). The PA2200 dielectric plates used in this experiment were $3 \mathrm{D}$ printed and as such bear a porous structure.

In addition to dielectric barrier material, the thickness of barriers is investigated as well. Investigated thicknesses were 1,2 , and $3 \mathrm{~mm}$ of silicone rubber and 1, 2, and $4 \mathrm{~mm}$ of 3Dprinted PA2200 plate. As for the Kapton based actuators, the barrier was made out of layers with a nominal thickness of $50 \mu \mathrm{m}$ with $39 \mu \mathrm{m}$ of additional adhesive. Total thickness of each layer was $89 \mu \mathrm{m}$. Actuators of 2, 4, 6, and 11 layers of Kapton were constructed with thickness of $0.18,0.36,0.54$, and about $1 \mathrm{~mm}$, respectively.

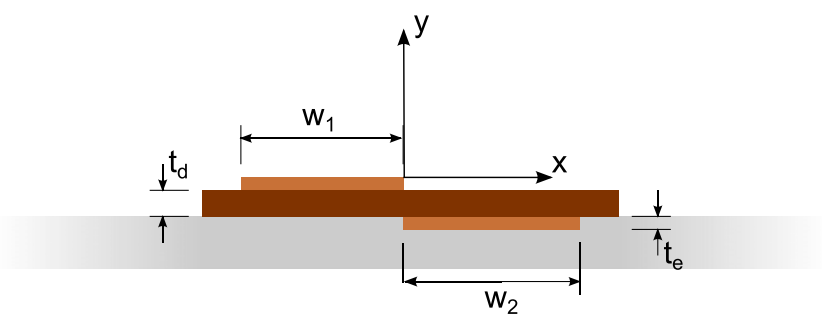

FIG. 1. Sketch of ns-DBD plasma actuator layout and Cartesian coordinate system. 
Other geometrical actuator characteristics were identical. Length of the copper electrodes was $14 \mathrm{~cm}$, width $5 \mathrm{~mm}$, and the electrode overlap was zero in all the tested cases. The thickness of the self-adhesive electrodes was $60 \mu \mathrm{m}$ with additional $40 \mu \mathrm{m}$ of adhesive. An overview of the geometrical proprieties of the tested actuator is listed in Table I.

Results are referenced to a Cartesian coordinate system where zero is set at the edge of the exposed electrode for all the tested cases, as shown in Figure 1.

The discharge was driven by a fast pulse solid-state power generator (FID), delivering $12.5 \mathrm{kV}$ nominal pulse voltage at frequencies up to $10 \mathrm{kHz} .^{8-10}$ The pulse rising time was approximately $3 \mathrm{~ns}$ (from $10 \%$ to $90 \%$ of the maximum voltage), while the pulse width was approximately $23 \mathrm{~ns}$. The power generator was connected to the actuator by means of a high voltage coaxial cable (type RG-217) $20 \mathrm{~m}$ long, of $50 \Omega$ impedance. The covered electrode was connected to the high voltage output, while the exposed was kept at ground potential.

A Tektronics AFG3252 Arbitrary Function Generator (AFG) was used for controlling the discharge given by a power generator, enabling regulation of trigger delay, number of pulses, and frequency for single pulse discharge mode as well as for bust discharge mode.

\section{Selection of materials}

The main motivation of this study is the elucidation of the effect of dielectric material on the characteristics of nsDBD plasma actuation. As such, the selection of the materials used as dielectric barrier for this parametric study intended to give as large range of dielectric and thermal properties as possible.

As suggested for AC-DBD, ${ }^{17,18}$ using a resistive barrier (typical values of resistivity range between $10^{8}$ and $\left.10^{9}[\Omega][\mathrm{m}]\right)$ instead of pure dielectric could help reducing the self-limiting effect typical of DBDs, thus increasing efficiency of the discharge. The extension of such argument to the case of ns-DBD is not trivial due to the different working principles. For the current study, three materials of disparate dielectric and thermal properties have been chosen in order to accentuate the effect of

TABLE I. Plasma actuator characteristics.

\begin{tabular}{lcccc}
\hline \hline & & \multicolumn{3}{c}{ Material } \\
\cline { 3 - 5 } Symbol & Description & Kapton & Silicone-rubber & PA2200 \\
\hline $\mathrm{W}_{1}$ & $\begin{array}{c}\text { Exposed electrode } \\
\text { width [mm] }\end{array}$ & 5 & 5 & 5 \\
$\mathrm{~W}_{2}$ & $\begin{array}{c}\text { Covered electrode } \\
\text { width [mm] }\end{array}$ & 5 & 5 & 5 \\
$\mathrm{t}_{\mathrm{d}}$ & $\begin{array}{c}\text { Dielectric thickness } \\
{[\mathrm{mm}]}\end{array}$ & 0.089 & $1 / 2 / 3$ & $1 / 2 / 4$ \\
$\mathrm{t}_{\mathrm{e}}$ & $\begin{array}{c}\text { Electrode thickness } \\
{[\mathrm{mm}]}\end{array}$ & 0.1 & 0.1 & 0.1 \\
$\mathrm{l}$ & $\begin{array}{c}\text { Actuator length } \\
{[\mathrm{mm}]}\end{array}$ & 140 & 140 & 140 \\
$\mathrm{~V}_{\mathrm{p}}$ & $\begin{array}{c}\text { Pulse voltage } \\
{[\mathrm{kV}]}\end{array}$ & 12.5 & 12.5 & 12.5 \\
\hline \hline
\end{tabular}

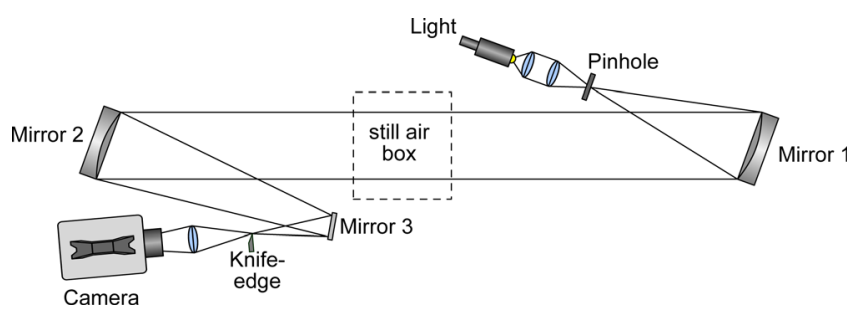

FIG. 2. Schematics of Schlieren experimental set-up arrangement.

material on the performance of these actuators. The three materials are Kapton, PA2200, and silicone rubber, exhibiting strong dielectric, weak dielectric, and resistive characteristics, respectively.

\section{Schlieren imaging}

Time-resolved Schlieren measurements were carried out in order to visualise and quantify the thermalized area resulting from ns-DBD actuation. A 12-bit CCD 4 Megapixel $(2016 \times 2016$ pixel $)$ La Vision Imager pro HS 4 M camera was used. The camera allowed measurements at $1 \mathrm{kHz}$ full frame, with an exposure time of $2 \mu \mathrm{s}$. Synchronization with the pulsed light source was achieved using a LaVision high-speed controller. A beamer Arc Lamp (Solarc, Lighting Technology) was used as light source of about $50 \mathrm{~W}$ power. The Schlieren setup was arranged in $\mathrm{Z}$ configuration. Magnification was about 0.2 , while the field of view (FOV) was approximately $11 \times 11 \mathrm{~cm}^{2}$. A spatial filter was configured to provide for density gradient along the vertical axis. A sketch of the arrangement for the Schlieren experiments is shown in Figure 2.

\section{Power measurements}

Power measurements were done via the back-current shunt technique in order to calculate energy associated with a single pulse. ${ }^{6,7,10,11,19}$ A shunt monitor resistor was built using 16 resistors, of $3.2 \Omega$ each, placed in parallel, resulting in an overall shunt resistance of $0.2 \Omega$. The shunt resistor was placed in the middle of the ground electrode of a $20 \mathrm{~m}$ long high voltage coaxial cable (type RG-217). This high voltage cable was used to deliver the high voltage nanosecond pulse from a power generator to the tested plasma actuator. A block scheme of the electrical configuration is given in Figure 3.

Measurements of pulse energy were performed by measuring the voltage across the shunt resistor directly with an oscilloscope. The application of a high voltage nanosecond

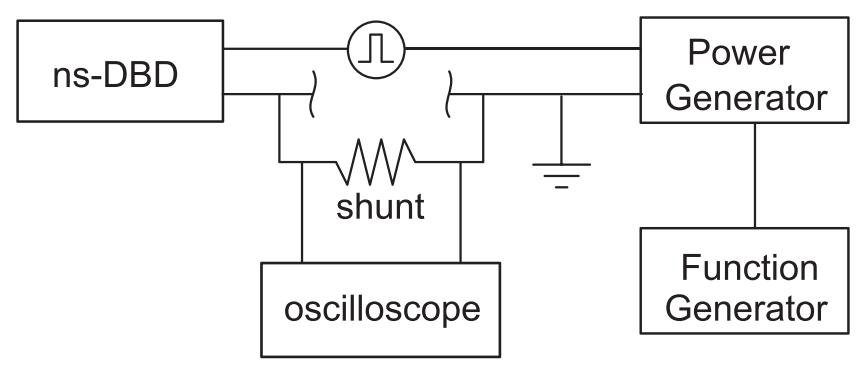

FIG. 3. Block scheme of the electric configuration of the back current shunt technique implemented for the measurements. 
pulse from a generator can be observed as a scaled voltage pulse over the shunt, measured by the oscilloscope. This can be used to calculate the energy input into the tested plasma actuator. Subsequently, a second negative peak was observed corresponding to the energy reflected back into the high voltage cable after the barrier discharge. Using these voltage signals, it was possible to calculate the current flowing through the shunt resistor and the power of each single pulse. According to transmission line theory, ${ }^{20}$ energy contained into each single nanosecond pulse can be calculated from

$$
E=\int P_{i n} d t=\int V_{\text {in }} \times I_{\text {in }} d t=\int \frac{V_{\text {in }}^{2}}{Z} d t
$$

where $V_{\text {in }}$ and $I_{\text {in }}$ are, respectively, the voltage and the current input, and $\mathrm{Z}$ is the impedance of the high voltage cable. ${ }^{21,22}$ It is noted that $V_{\text {in }}$ was not directly measured but calculated from the voltage developed across the shunt $\mathrm{V}_{\mathrm{sh}}$ according to

$$
V_{\text {in }}=V_{s h} \times K_{s h} .
$$

In this way, the total energy transferred to the flow and dissipated by the plasma actuator was calculated as the difference between the absolute value of the energy given by the first peak of the voltage signals $\left(E_{i n}\right.$, the energy input) and the second peak of the voltage signals $\left(\mathrm{E}_{\text {out }}\right.$ the energy reflected back into the high voltage cable), according to

$$
E_{\text {ep }}=E_{\text {in }}-E_{\text {out }},
$$

where $E_{e p}$ is the energy per pulse transferred to the flow and dissipated within the plasma actuator itself.

\section{Calibration of back-current shunt technique}

A technique to measure electrical energy by a ns-DBD plasma actuator is the back-current shunt technique. ${ }^{6,10,11,19}$ This technique relies on the measure of incident and reflected currents through the actuator circuit. Calibration of the shunt monitor is performed by applying a known voltage signal through the actuator circuit and measuring the voltage across the shunt resistor. The ratio between the voltage input and output $\left(\mathrm{K}_{\mathrm{sh}}\right)$ should be very close to the ratio of cable impedance and shunt resistance

$$
K_{s h}=\frac{Z}{R_{s h}}=\frac{V_{a}}{V_{s h}},
$$

where $\mathrm{Z}$ is the impedance of the cable, $\mathrm{R}_{\mathrm{sh}}$ is the shunt resistance, $\mathrm{V}_{\mathrm{a}}$ is the applied known voltage signal, and $\mathrm{V}_{\mathrm{sh}}$ is the voltage reading across the shunt. For the current study, the shunt resistor was calibrated by applying a known signal of $10 \mathrm{~V}$ from an Arbitrary Function Generator (Tektronics AFG3252) and measuring the voltage across the shunt directly with a digital oscilloscope of $1 \mathrm{GHz}$ (set at matched impedance to the high voltage cable). A $20 \mathrm{~dB}$ attenuator was used in order to protect the oscilloscope from voltage overload. The attenuation constant of the shunt resistor $\mathrm{K}_{\mathrm{sh}}$ is theoretically calculated to be 250 using

$$
K_{s h}=\frac{Z}{R_{s h}},
$$

where $\mathrm{Z}=50 \Omega$ is the impedance of the high voltage cable and $R_{\mathrm{sh}}=0.2 \Omega$ is the total resistance of the shunt. The calibration process yielded a $K_{\text {sh }}$ of 253.97 , value very close to the one calculated.

\section{Discharge light intensity}

Time-averaged light intensity of the plasma discharge was measured with the use of a 10.2 Megapixel $(2872 \times 2592$ pixel) Nikon D80 digital camera with CCD sensor. The magnification was 0.05 and the FOV $46 \times 31 \mathrm{~cm}^{2}$. Images were taken with an exposure time of $1 \mathrm{~s}$. Before every discharge imaging, a background image was taken in order to ensure only discharge-associated light would be used in calculations. The camera sensitivity was set to ISO 400 and the diaphragm to an $\mathrm{f}^{\#}$ of 3.8.

\section{Material tests}

Measurements of dielectric constant, electrical volume resistivity, and thermal conductivity of the tested materials were carried out in order to qualify actuator performance as function of proprieties of the materials used for the barrier. All tests were performed in a controlled environment so to keep constant boundary conditions, therefore reducing the uncertainties of the measurements.

The relative permittivity or dielectric constant $\epsilon_{r}$ was determined by measuring the capacitance $(\mathrm{C})$ of a circular parallel plate capacitor using a dielectric barrier made out of the tested material. The dielectric constant $\epsilon_{r}$ can be determined when knowing the absolute dielectric permittivity of vacuum $\left(\epsilon_{0}=8.854 \times 10^{-12}[\mathrm{~F}] /[\mathrm{m}]\right)$, the barrier thickness $\left(\mathrm{t}_{\mathrm{d}}\right)$, and electrode overlap area $(\mathrm{A})$ using

$$
C=\epsilon_{0} \times \epsilon_{r} \times \frac{A}{t_{d}} .
$$

The test samples were made out of punched discs of $20 \mathrm{~mm}$ diameter of the tested material on which gold electrodes of $10 \mathrm{~nm}$ thickness on either side were sputtered using a Balzers SCD 040 sputtering device. These electrodes were $20 \mathrm{~mm}$ diameter on one side and $14 \mathrm{~mm}$ on the other side so to avoid short circuits over the sides of the material sample.

An Agilent 4263B LCR Meter was used at a frequency of $1 \mathrm{kHz}$ and a voltage of $1 \mathrm{~V}$, having an accuracy of $0.01 \times 10^{-12} \mathrm{~F}$, to measure the capacitance. The probes of this machine were connected to the sputtered gold electrodes of the test samples. All thicknesses were tested and ensemble averaged values are reported in Table II. Moreover, thermal conductivity was measured on samples without electrodes through a C-Therm Technologies TCiTM thermal conductivity system, which applies a modified transient plane source technique. ${ }^{23} \mathrm{~A}$ small temperature rise was applied followed by a cooling down period to measure the thermal conductivity of the material. Electrical volume resistivity was calculated by measuring displacement current induced by a high DC voltage through the tested materials via electrodes of $40 \mathrm{~mm}$ diameter. Resistance (R) of the tested materials was then calculated and converted in electrical volume resistivity using the formula 
TABLE II. Tested dielectric materials properties.

\begin{tabular}{|c|c|c|c|c|}
\hline \multirow{2}{*}{ Symbol } & \multirow{2}{*}{ Description } & \multicolumn{3}{|c|}{ Material } \\
\hline & & Kapton & Silicone-rubber & PA2200 \\
\hline $\mathrm{Vr}$ & Dielectric strength $[\mathrm{kV}] /[\mathrm{mm}]^{\mathrm{a}}$ & 134.8 & 19.7 & 16 \\
\hline$\varepsilon_{\mathrm{r}}$ & Dielectric constant $\left[\mathrm{C}^{2}\right] /[\mathrm{N}]\left[\mathrm{m}^{2}\right]$ & 2.9 & 3.0 & 3.6 \\
\hline K & Thermal conductivity $[\mathrm{W}] /[\mathrm{m}][\mathrm{K}]$ & 0.29 & 0.27 & 0.1 \\
\hline$\rho$ & Electrical volume resistivity $[\Omega][\mathrm{m}]$ & $2.97 \times 10^{9}$ & $3.41 \times 10^{8}$ & $4.0 \times 10^{8}$ \\
\hline $\mathrm{c}$ & Specific heat $[\mathrm{J}] /[\mathrm{g}][\mathrm{K}]^{\mathrm{a}}$ & $4.1 \times 10^{-3}$ & $6.9 \times 10^{-3}$ & $4.8 \times 10^{-3}$ \\
\hline
\end{tabular}

${ }^{\mathrm{a}}$ These values are obtained from manufacturer data sheets.

$$
\rho=R \times \frac{A}{t_{d}},
$$

where $A$ and $t_{d}$ are area and thickness of the tested barrier, respectively.

\section{RESULTS}

\section{Materials properties}

Table II presents an overview of measurements of electric and thermal characteristics of the three materials used for this investigation. Moreover, the data set is completed reporting data from material manufacturer, i.e., for dielectric strength. The dielectric constant is assumed independent of the thickness of the material. It is noted that the reported values for electrical volume resistivity are the one corresponding to the thinnest tested barrier per each material (also reported in Figure 4).

It is observed that Silicone and PA2200 show a smaller dielectric strength with respect Kapton. However, a discharge produced using these materials still results in a glow discharge, which is typical for a dielectric barrier. ${ }^{24}$

In Figure 4, results are reported for the calculated electrical volume resistivity per each investigated barrier.

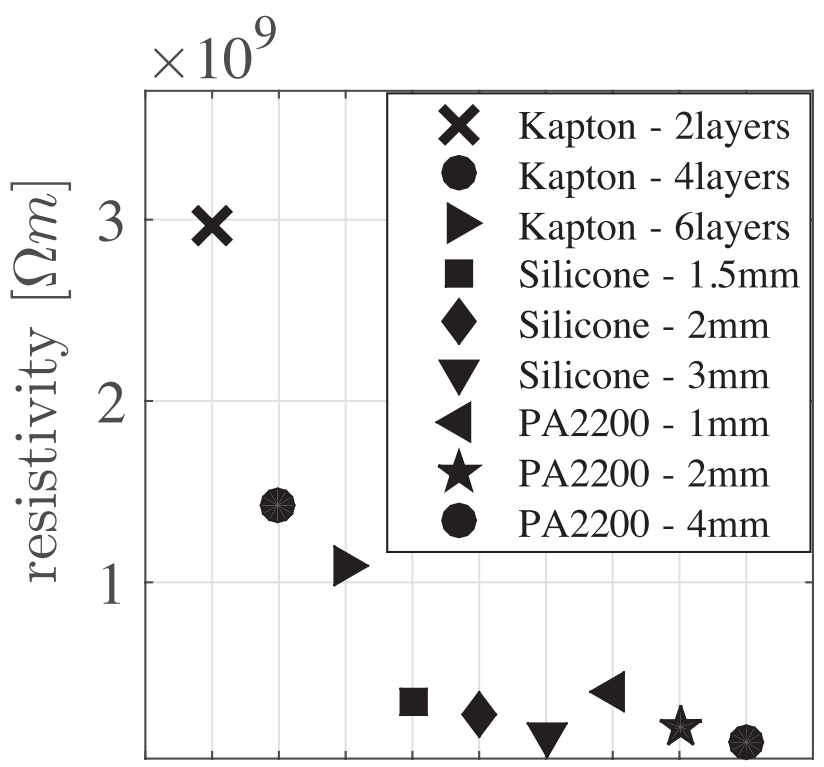

FIG. 4. Electrical volume resistivity of all the tested barriers. Labels on the $\mathrm{X}$-axes indicate Kapton $(\mathrm{K})$ with thickness in number of layers (i.e., 2, 4, and 6), silicone-rubber (S), and PA2200 (P) with thickness in millimetres.
Results show that volume resistivity is affected by the material thickness. This is due to impurities present in thicker barriers, i.e., more layers of Kapton tape include more layers of silicone-based adhesive. For this reason, calculations based on the thinnest barrier are considered more reliable and reported in Table II.

\section{Electrical power characteristics}

In Figure 5, the voltage input per single pulse is presented. The three plots show the actual nanosecond voltage pulse, up to $12 \mathrm{kV}$ (nominal, first peak), and the pulse reflected back (second peak). The first peak in each plot is proportional to the amount of energy given per pulse, while the second peak is proportional to the energy that is reflected back. Therefore, the difference between the two peaks gives an indication of how much energy was used by the plasma actuator per each pulse. However, it must be pointed out that the used energy is composed of the energy transferred to the flow and the energy dissipated internally into the barrier of a plasma actuator.

The voltage reflected back increases as the thickness of the dielectric barrier increases, regardless of the material. This result indicates the energy used by the actuator is inversely proportional to the thickness of the dielectric barrier. This behaviour is due to the fact that the thicker is the dielectric barrier the further away the two electrodes are in the vertical direction. Therefore, for a thicker dielectric barrier, a weaker electrical field can be generated between the two electrodes of a plasma actuator, delivering a correspondingly weaker discharge.

Although direct voltage measurements give a qualitative indication of the electrical energy budget of the actuation, non-optimised components in the power supply contribute to inaccuracies, which prohibit the direct evaluation of transferred energy. In order to rectify this, accurate energy measurements are performed using the back-shunt current technique. In Figure 6, the voltage input of a single pulse acquired via back-current shunt technique was compared for the three different materials under investigation, at fixed thickness of $1 \mathrm{~mm}$. Due to the impedance mismatch between the shunt and the voltage attenuators used to log the signals directly to an oscilloscope, some energy is lost. Nevertheless, the total energy per pulse $E_{e p}$, i.e., the sum of energy transferred to the flow and energy dissipated into the actuator can still be safely calculated as the difference between the energy given as input and the energy reflected back into the high 


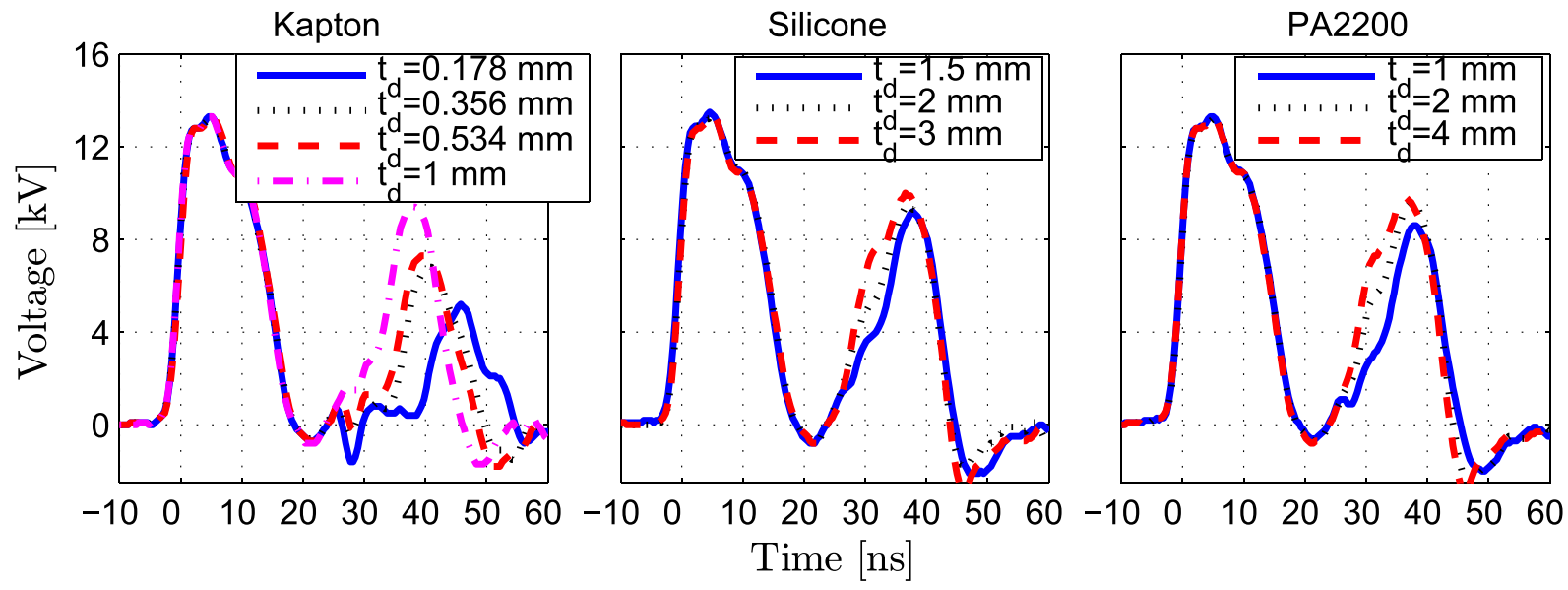

FIG. 5. Voltage measurements. Effect of different barrier thickness per each tested material.

voltage cable. Values of the calculated energy are given in Figure 6.

In Figure 6, it is observed that for a fixed thickness, the amount of energy that is reflected back into the high voltage cable is not the same, even if the energy input is exactly the same. However, the reflected energy is in the same order of magnitude for all the cases. Thus, from Figure 6, it is evident that for the cases of Kapton, less energy is transferred to the actuator. This behaviour is attributed to the different electrical characteristics of the material used for the study. In Table II, it is reported that Kapton has a smaller dielectric constant than the other two tested materials and thus, according to Eq. (7) for a fixed thickness, also a smaller capacitance. It is evident that the fraction of energy stored or dissipated in the material is increased with increasing actuator capacitance and decreasing material resistivity. This competes with the amount of energy actually released into

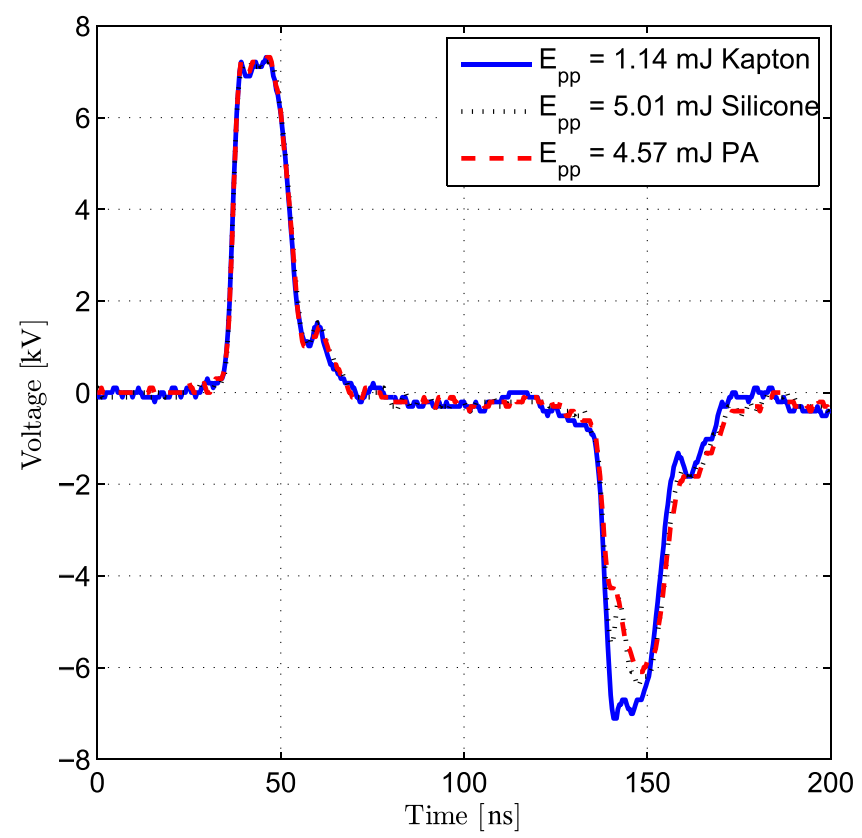

FIG. 6. Voltage measurements using back-current shunt technique. Comparison of three different materials with approximately the same thickness (about $1 \mathrm{~mm}$ ). the flow. A comparison with energy per pulse reported in literature is done and reported in Table III.

As can be seen from Table III, ${ }^{25,26}$ the energy per pulse for Kapton-based actuators is inversely proportional to the thickness of the dielectric barrier.

\section{Discharge imaging}

A comparison between the light intensity produced by a discharging nanosecond plasma actuator is shown in Figure 7, having a dielectric barrier at approximately the same thickness of about $1 \mathrm{~mm}$, for the three different materials under investigation. The $x=0$ indicates the edge of the exposed electrode where discharge starts from, as illustrated in Figure 1.

The mean light intensity was calculated over a span of $5 \mathrm{~cm}$ in the middle of the actuator. The comparison was done at two different frequencies, i.e., 0.1 and $1 \mathrm{kHz}$. Such experiment was aimed to observe differences into the plasma layer morphology, i.e., different length and intensity or distribution of the plasma filaments.

It is evident that the frequency of discharge affects the mean light emission. The light intensity is proportional to the pulse frequency for all three dielectric barrier materials. It is interesting to note that in the case of Silicone and PA, plasma discharge is forming also on the opposite side with respect to the location of the covered electrode. In Figure 7, two peaks are observed: one above the exposed electrode for all the three materials and a second one around $\mathrm{x}=-5 \mathrm{~mm}$

TABLE III. Values given in literature for ns-DBD plasma actuators made out of Kapton tape and a peak pulse voltage of about $10 \mathrm{kV}$ input.

\begin{tabular}{lcc}
\hline \hline Authors & Barrier thickness & Pulse energy per unit length \\
\hline Dawson and Little & $0.075 / 0.15 / 0.25 \mathrm{~mm}$ & $0.83 / 0.69 / 0.56 \mathrm{~mJ} / \mathrm{cm}$ \\
Benard et al. $^{12}$ & $0.152 \mathrm{~mm}$ & $0.3 \mathrm{~mJ} / \mathrm{cm}$ \\
Starikovskii et al. $^{19}$ & $0.3 \mathrm{~mm}$ & $0.4 \mathrm{~mJ} / \mathrm{cm}$ \\
Correale et al. $^{6,10}$ & $0.267 \mathrm{~mm}$ & $0.56 \mathrm{~mJ} / \mathrm{cm}$ \\
Takashimaa $^{11}$ & $0.3 \mathrm{~mm}$ & $0.3 \mathrm{~mJ} / \mathrm{cm}$ \\
Ndong $^{26}$ & $0.3 \mathrm{~mm}$ & $0.2 \mathrm{~mJ} / \mathrm{cm}$ \\
Little et al. & & $0.36 / 0.21 \mathrm{~mJ} / \mathrm{cm}$ \\
This study & $0.27 / 0.44 \mathrm{~mm}$ & $0.08 \mathrm{~mJ} / \mathrm{cm}$ \\
\hline \hline
\end{tabular}



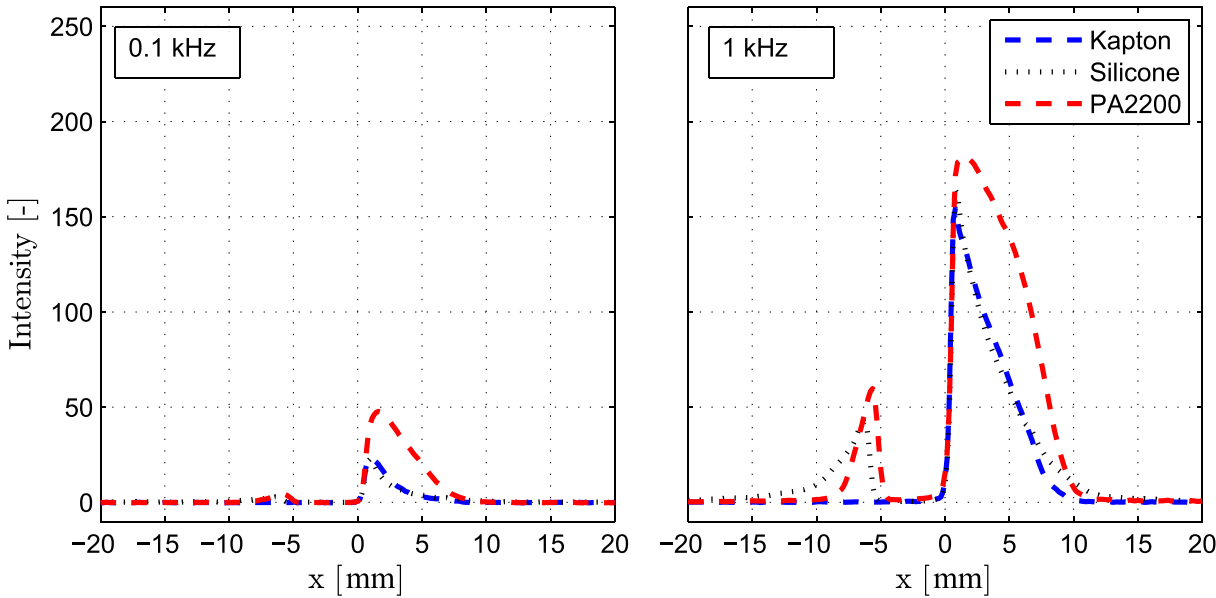

FIG. 7. Comparison of mean light intensity of the discharge for different materials with fixed thickness for two different frequencies of discharge. for the cases of Silicone and PA. This is attributed to lower volume resistivity of these materials compared to Kapton (see Table II). This allows electrons to run through the barriers more freely so to diffuse in every direction where a lower electrical potential is felt. Therefore, those barriers cannot prevent the discharge from occurring on the opposite side of the exposed electrode with respect to the side where the covered electrode is located. This result also indicates that for such materials, the energy is deposited over a larger region, i.e., at every location where discharge takes place. Furthermore, it is noted that the mean light intensity by a discharging plasma actuator reaches higher peaks when the dielectric barrier is PA. This is further verified observing the actual discharge in Figure 8.

Figure 8 shows the actual discharges for the three materials under investigation. In these tests, the dielectric barrier was always $1 \mathrm{~mm}$ thick and the frequency of discharge was $1 \mathrm{kHz}$ and maximum allowable voltage, i.e., $10 \mathrm{kV}$. The different streamer morphologies are clearly visible. As the images show, the discharge is filamentary independently from the material of the dielectric barrier. However, in case of silicone-rubber, a more distributed discharge is visible, with smaller filaments and a very bright edge of the exposed electrode. The case of Kapton looks very similar to the former case, although larger and more localized discharge filaments are visible. In case of PA2200, a different discharge is observed. The filaments are elongated and more localised with respect to the former two cases. This behaviour is due to the porosity of the material.

\section{Energy deposition characteristics}

Due to strong thermal effects associated with ns-DBD, Schlieren technique was selected in order to qualify gas density variations. Such approach is similar to the one followed in previous works. ${ }^{7,8,10-12}$ Although several investigations were focused on the effect of ns-DBD actuators in quiescent conditions, ${ }^{12,25,26}$ little attention was given to the role of the material of the barrier for ns-DBDs in the energy deposition process. Towards this goal, a qualification investigation on the total energy discharged and deposited into the flow based upon number of nanosecond pulses is carried out.

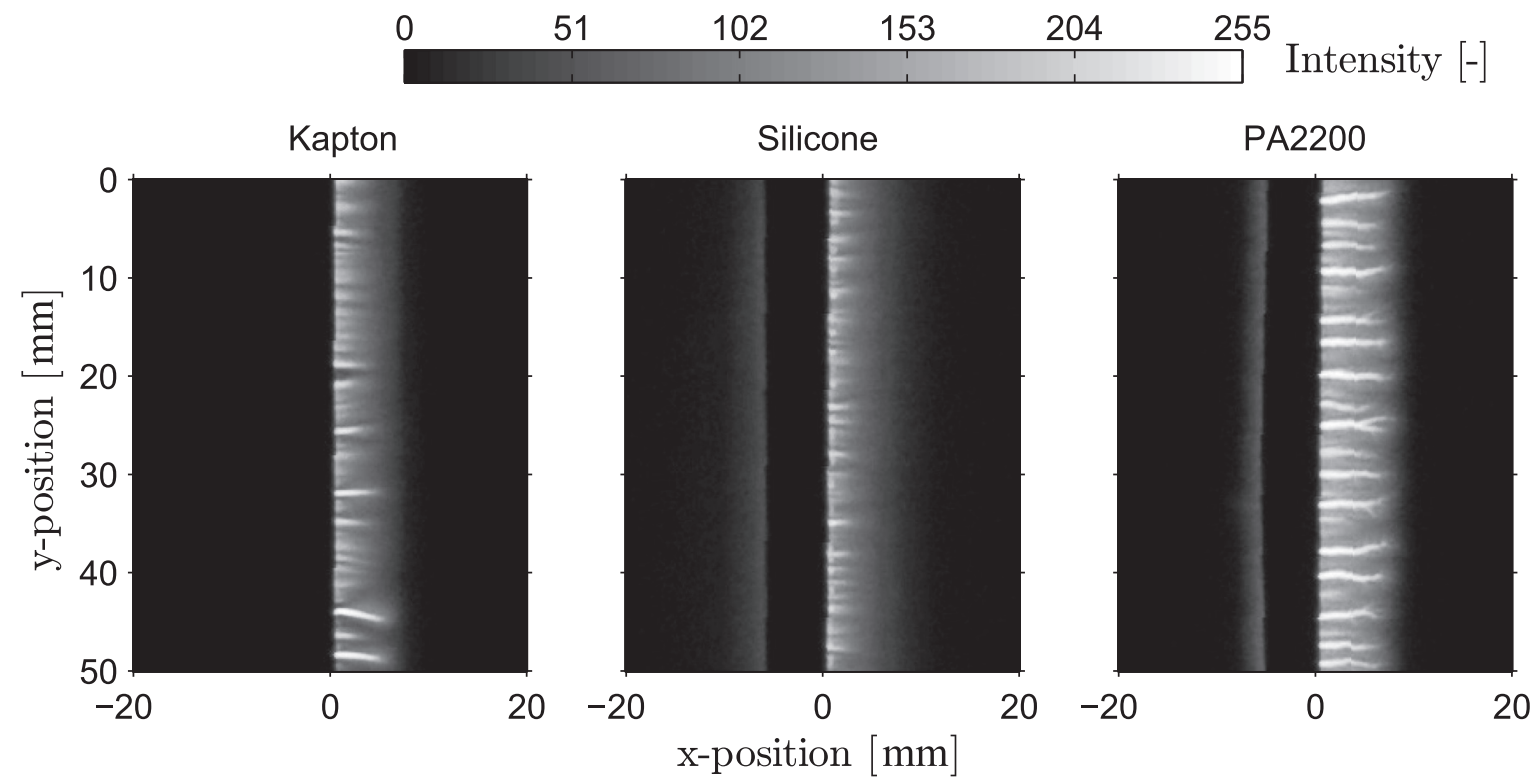

FIG. 8. Light measurements. Comparisons of actual discharge for different materials and the same thickness at $1000 \mathrm{~Hz}$ discharge frequency. A colour bar gives the levels of the actual intensity. 


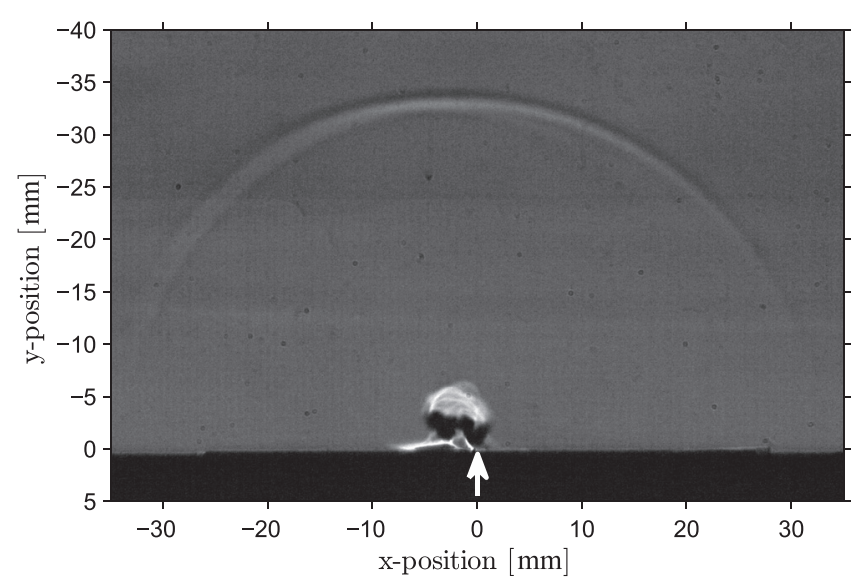

FIG. 9. Schlieren imaging for quiescent flow, 50 pulses, $10 \mathrm{kV}, 10 \mathrm{kHz}$. Dielectric barrier is made out of two layers of Kapton tape (see Table I). Image taken $5 \mathrm{~ms}$ after the beginning of the discharge. The white arrow indicates the location of the electrode gap.

The experiments were all carried out with a discharge frequency of $10 \mathrm{kHz}$. Number of pulses investigated varied from 10 to 100 pulses with steps of 10 pulses.

Figure 9 shows typical compression waves at a time instant $5 \mathrm{~ms}$ after the beginning of the discharge of a burst of 50 pulses at $10 \mathrm{kHz}$ in the quiescent flow condition. The compression wave and the following thermal effect are clearly visible as observed previously. ${ }^{9,10}$

After a pulsed nanosecond discharge, a rapid increase in gas temperature within the discharge volume is occurring. As the gas does not have the time to expand, a cylindrical compression wave is formed originating at each streamer location. ${ }^{7}$ The shockwave shown in Figure 9 was an envelope in spanwise direction of all the cylindrical shockwaves produced by each streamer of the glow discharge. As reported by Benard et al., ${ }^{12}$ a shockwave is formed for both the rise and the fall parts of the voltage signal. However, the power generator used for the current test produces a voltage pulse so short (about 20 ns) that into the Schlieren image (Figure 9) the two shockwaves look to be merged together.

It is important to notice that a Schlieren image depicts changes in density along the axes perpendicular to the direction of the knife, i.e., y-axis for this study. Such density gradient is associated with a change of temperature due to the energy discharged by the plasma actuator. ${ }^{9,10}$
As shown in the left-hand side of Figure 10, a region of high temperature forms a gradient of density that can be observed with Schlieren technique in the vicinity of the discharge region. Moreover, considering the extremely rapid deposition of energy in the air (order of microseconds after each pulse), a "hotspot" appears completely decoupled from the weak compression waves. It has been observed that each single pulse contributes to the formation of this "hotspot," and after the end of the discharge, thermal expansion takes place. $^{10,13}$

An estimate of the heated region is obtained via an inhouse image-processing algorithm, developed with Matlab, which isolates the region of interest in the image (where density gradient is located) based on a threshold criterion. Such threshold is set based on the average shade of grey of the image background, thus it is able to set a specific threshold accordingly to the light intensity of the image itself. Regions where a thermal gradient induces a density gradient can be isolated in this manner. Counting number of pixels contained within said isolated region and knowing pixel pitch and magnification of the experimental set-up, it is possible to calculate the heated area where energy of discharge is deposited. Moreover, it is possible to track the evolution of the said area in time. 9,10

As shown in the right-hand side of Figure 10, an example of the estimated area using the discussed image-processing strategy is given: The white region in this frame is an estimation of the heated gas volume created by the discharge.

This image-processing technique was applied to the Schlieren images of all the energy input cases investigated in order to evaluate the size of the heated area as a function of elapsed time from the beginning of the discharge. In Figure 11, trends of the estimated area are shown for the investigated barriers.

The material used for the dielectric barrier influences significantly the energy that is deposited within the discharge volume. When the dielectric barrier is made out of Kapton, the size of the area heated up by the electrical discharge is one order of magnitude higher than the one measured for the other two material investigated. Additionally, for silicone rubber and PA2200, results show a much smaller rate of energy deposition with respect to the Kapton case independently from the thickness of the dielectric barrier.
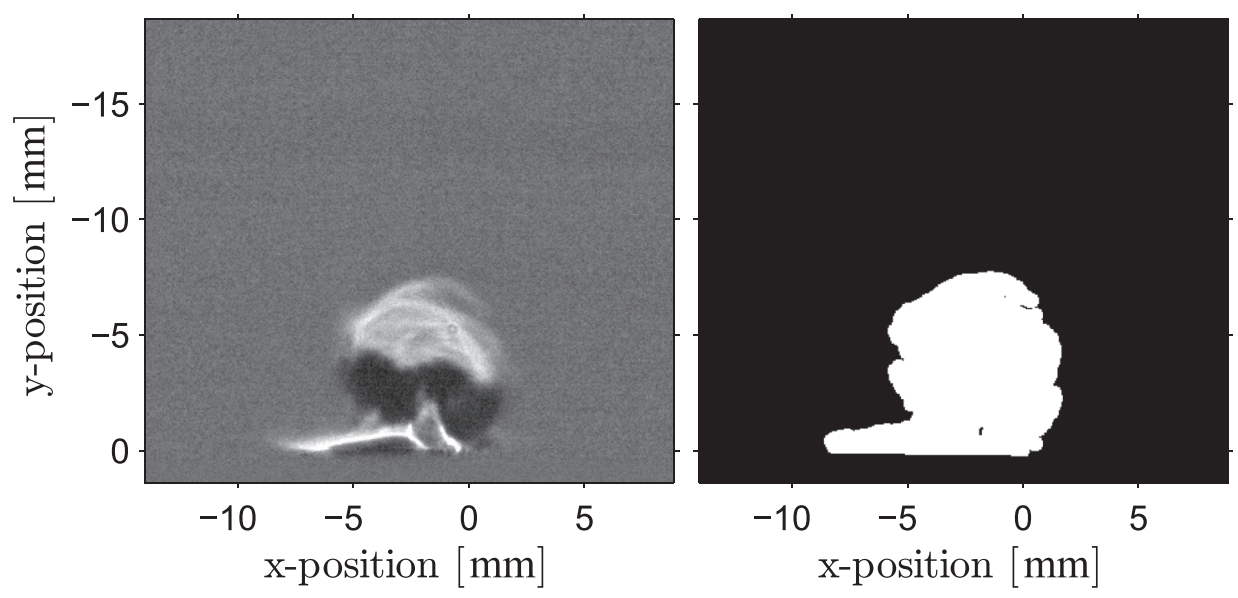

FIG. 10. Example of processing procedure. On the left-hand side, an unprocessed frame. On the right-hand side, the processed image showing the estimated area of the hot spot. 

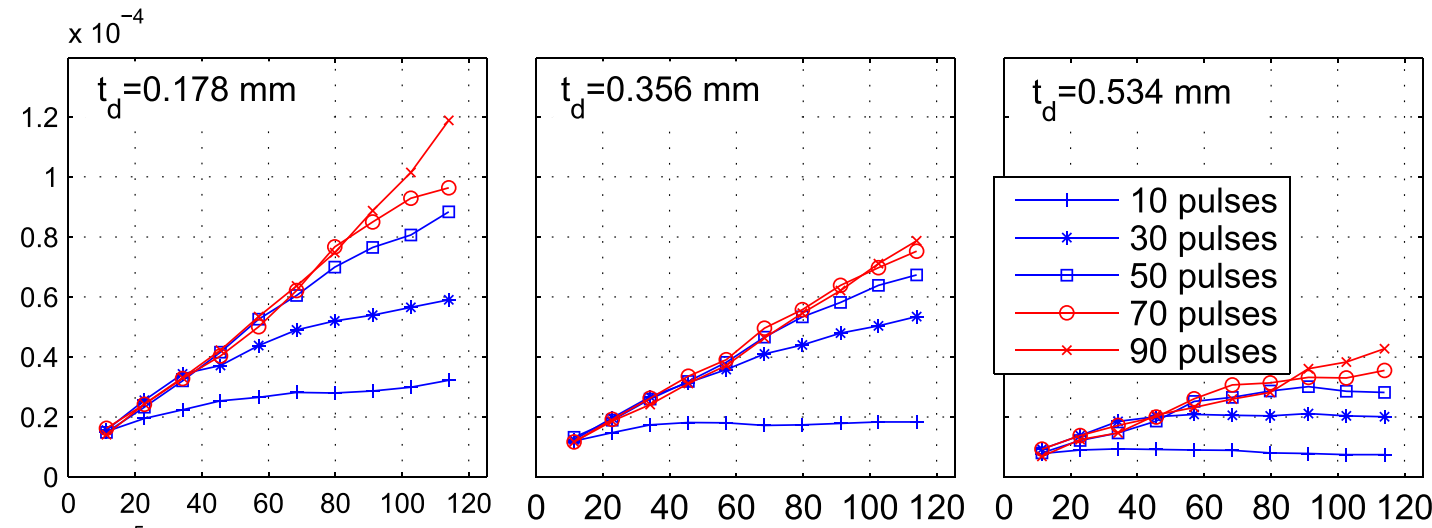

Kapton
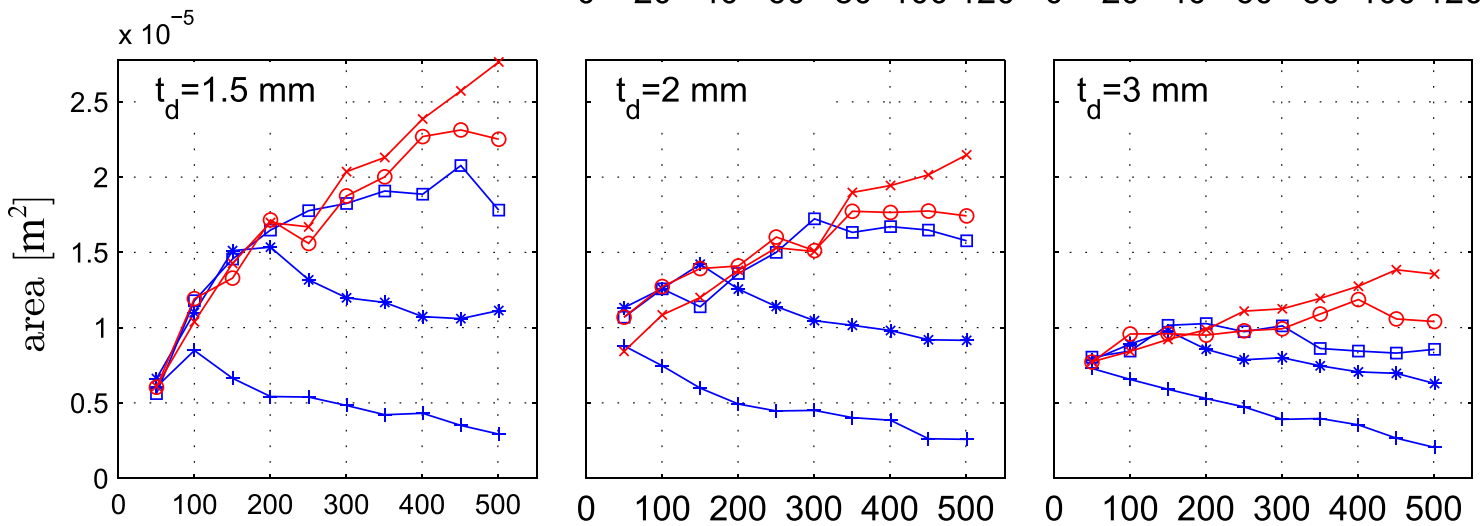

Silicone
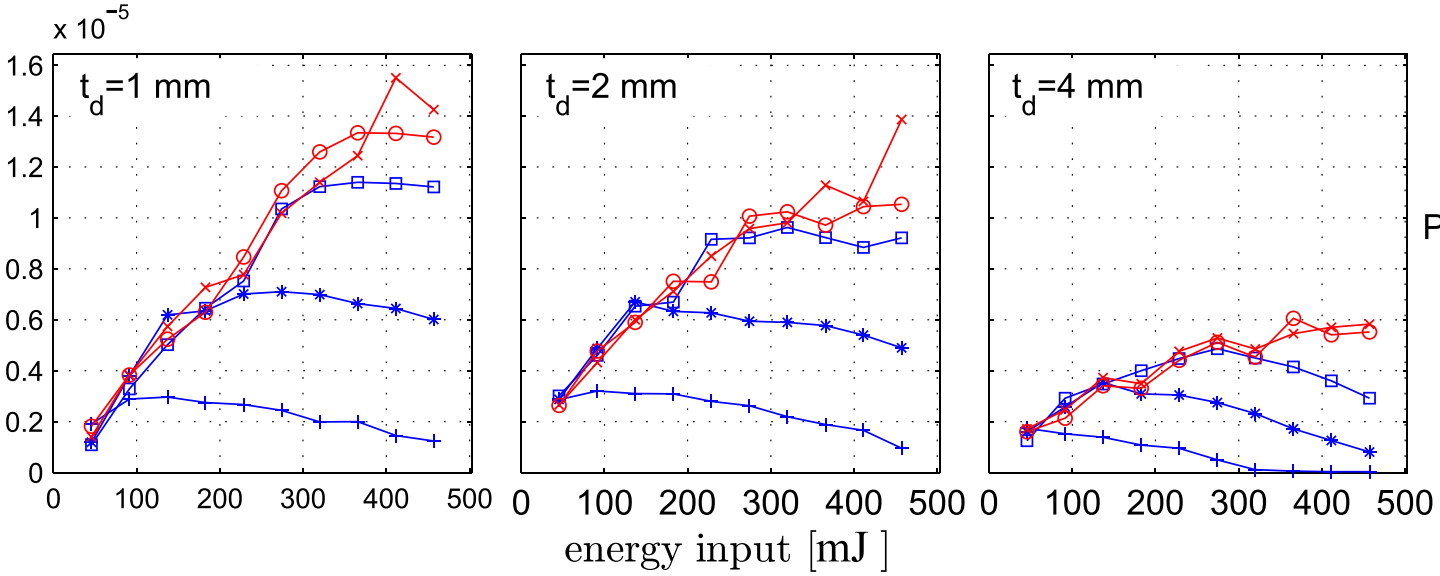

PA2200

FIG. 11. Estimated heated area evolution in time, for different energy inputs.

In Figure 12, the heated area is plotted as function of the energy input. It is noticed that for all the cases, a qualitative linear growth of the heated area is observed for as long as the discharge is on, ${ }^{10}$ which indicates that each pulse discharged contributes to the formation of residual heat which generates the observed density gradient. ${ }^{10,13}$

For thin dielectric barriers, results for PA 2200 show on the one hand estimated heated area in the same order of magnitude of the Silicone barrier, on the other hand, trends of energy deposition are linear for as long as the discharge is on (Figure 11). Nevertheless, the thicker the barrier becomes the smaller is the rate of energy deposited within the discharge volume (Figure 12). Such behaviour is observed independently from the material. This result suggests that for a thicker dielectric barrier, less energy is actually deposited in the discharge volume. ${ }^{26} \mathrm{~A}$ possible reason for this behaviour is the larger vertical distance between the electrodes dictated by a thicker barrier, thus a lower electric field developed between them. To a weaker electrical field corresponds a weaker discharge which results in a lower energy deposition. Moreover, the three investigated materials have a similar thermal conductivity and specific heat (see Table II). As such, the differences observed in Figures 11 and 12 are attributed to a larger thermal capacitance inherently possessed by a thicker barrier.

Considering that thermal expansion is function of the thermal gradient, which in turn is function of energy deposition, it must be noted that for different materials, a different energy deposition is achieved thus different thermal gradients, therefore direct comparison between materials for the same energy input is not possible. However, it is possible to determine which material performs best in terms of net energy deposited within the discharge volume and consequently which material dissipates internally the lowest amount of energy. 


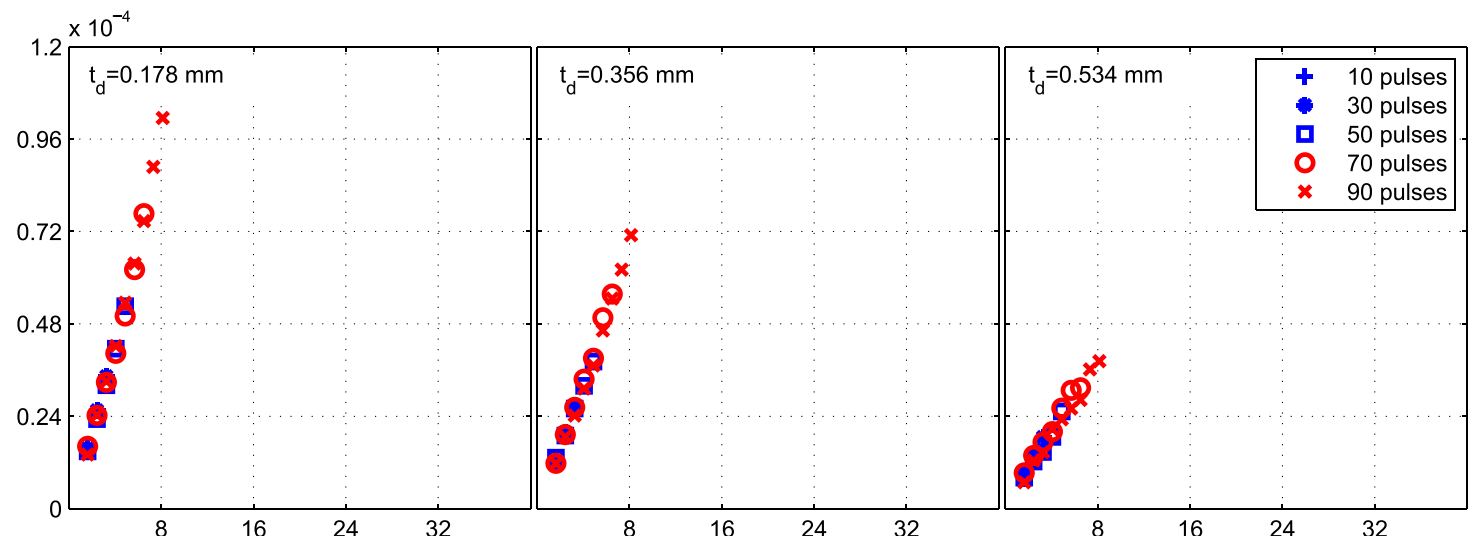

Kapton
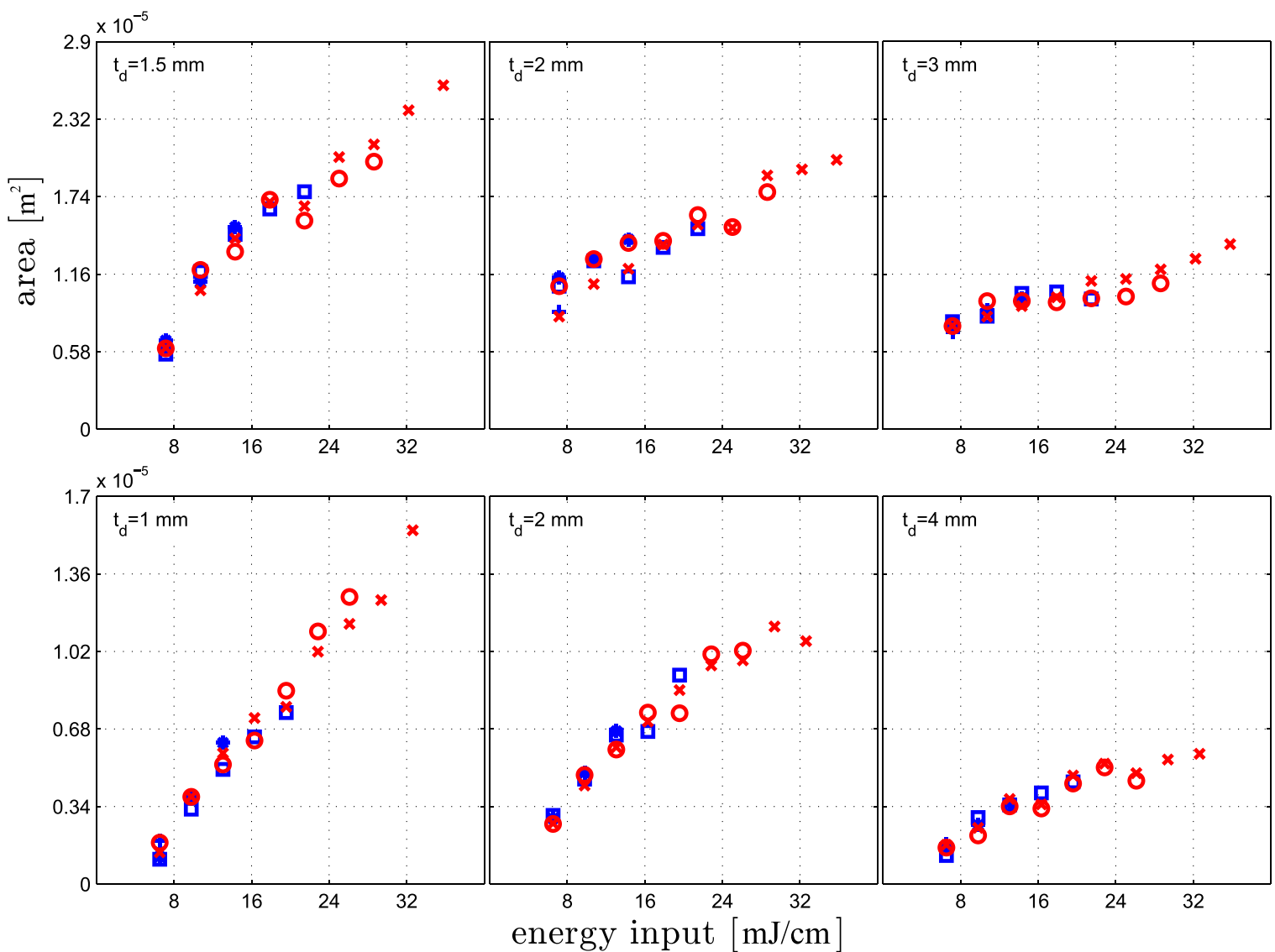

PA2200

FIG. 12. Estimated heated area as function of energy input.

Currently, quantification of density gradients based on the heated volume (measured with the method employed here), heat capacitance of air and total energy input is not possible due to the fact that it is impossible to distinguish between the amount of energy dissipated internally into the actuator and the amount of net energy deposited into the discharge volume. The authors are currently working on expanding the measurement technique to account for these factors. The evaluation of the different energy contributions is beyond the scope of this study.

In Figure 13, changes of the estimated heated area with respect to the thickness of the barrier were compared for three input energy levels $(10,50$, and 100 pulses). In accordance with the latter observation, it is observed that increasing the thickness of the barrier, the energy released into the air within the discharge volume always reduces for the case of
Kapton. ${ }^{26}$ As for the other two materials, a less regular behaviour is observed.

In Figure 14, the time evolution of estimated heated up areas scaled with the thickness of the barrier itself is compared for each one of the three tested materials with a fixed energy input, i.e., 30 pulses thus discharge on for $3 \mathrm{~ms}$ for a discharge frequency of $10 \mathrm{kHz}$. A linear increase in the relative estimated area is observed for as long as the discharge is on, demonstrating that independently from the material, each single pulse contributes of the same amount to the energy budget deposited within the discharge volume. However, the absolute values for Silicone and PA2200 are one order of magnitude lower than Kapton, which indicates that the energy dissipated internally within a barrier strongly depends on the material, i.e., heat capacity and/or dielectric strength. Moreover, the rate of energy dissipated must also present a 

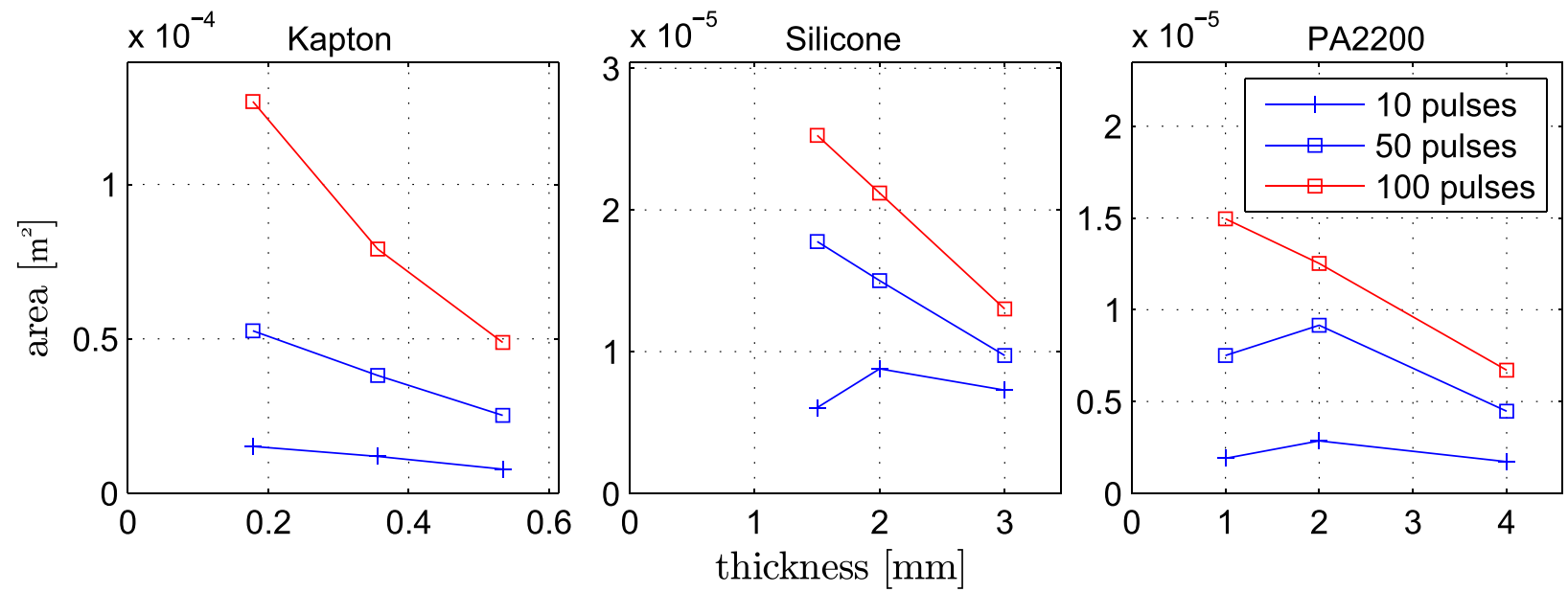

FIG. 13. Estimated area dependent on thickness of the dielectric barrier, per each tested material.

linear trend, considering that total energy input is constant for the three investigated cases. After the discharge is over, thermal expansion takes over and trends of time evolution of the heated up areas are not linear anymore (see also Figure 11). It can be seen that especially for the case of the silicone-rubber barrier, after the end of the discharge, the relative heated area reduces. The thermal conductivity measured for the three tested material is about the same order of magnitude (see Table II). As such, this behaviour is attributed to the fact that the thermal capacitance of the barriers is different. Thickness of those barriers made out of Silicone and PA is larger with respect to a barrier made out of Kapton. Therefore, thermal expansion is suppressed and heat is absorbed and dissipated within the barrier itself, actually cooling down the heated air. The linear behaviour that the curves show during the discharge demonstrates the dependency of the energy deposition rate relatively to thickness of the barrier, regardless of the material.

Such characteristic might be due to the higher electrical field produced when the dielectric barrier is thinner. In fact, a thinner dielectric barrier allows the two electrodes to lay closer to each other, thus increasing the electrical field, and so the intensity of the discharge accordingly.
Looking at Figures 6, 11, and 14, it is clear that the rate of energy transfer is lower for the cases of Silicone and PA and the relative estimated heated areas are one order of magnitude smaller than the respective results for Kapton. Therefore, it is given that for a fixed thickness, in case of a dielectric barrier made out of Kapton, more energy is transferred to the air rather than dissipated internally within the dielectric barrier. This signifies that the latter materials are less efficient than the former one in terms of energy deposition into the flow. Such behaviour is attributed to the lower volume resistivity of these materials (see Table II), which allows more current to flow throughout the barrier so increasing the energy losses.

\section{Calibration of the method}

To qualify a multiple-discharge energy deposition, as the ones reported in this paper, the classic shockwave intensity/radius vs time method was not suitable since each pulse within a burst has the same nominal voltage. A cumulative effect could not be observed using this method since each single shockwave is the same. Therefore, a method capable of capturing a cumulative effect of multiple pulses was implemented. Single pulses acquisition were carried out in
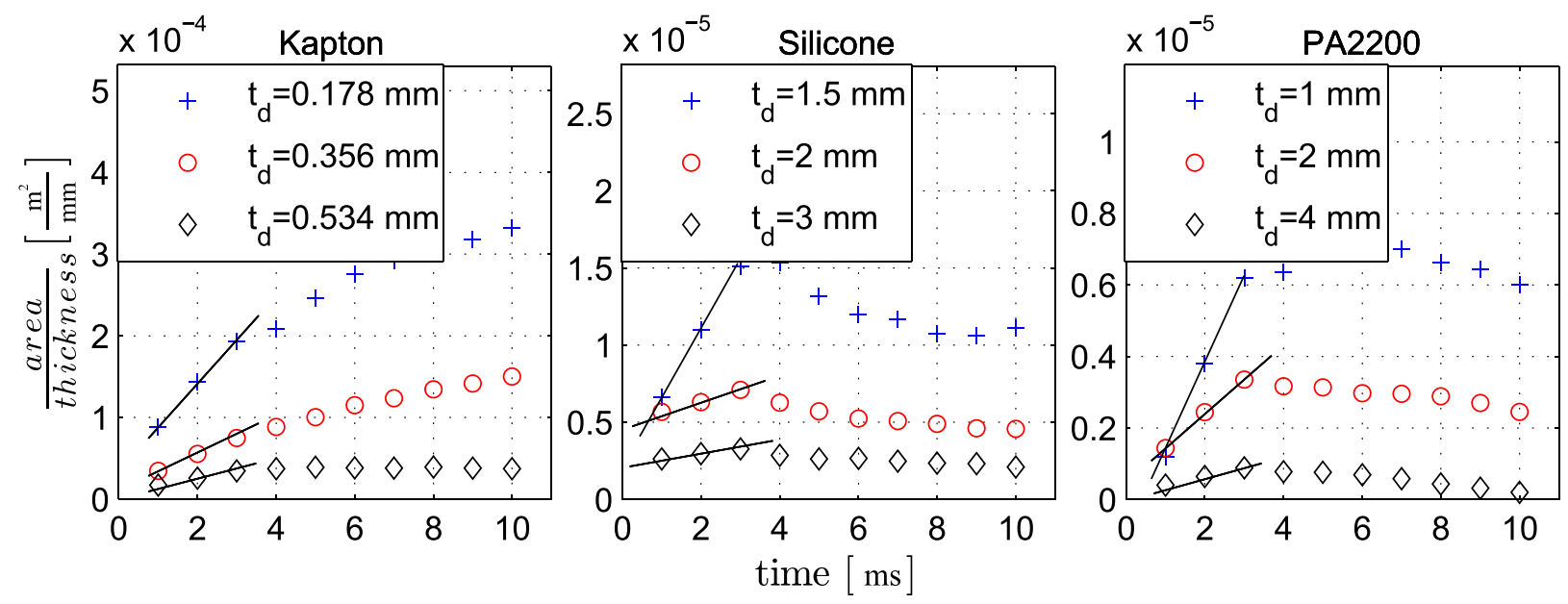

FIG. 14. Evolution in time of the estimated area scaled upon the thickness of the dielectric barrier, per each tested material. 

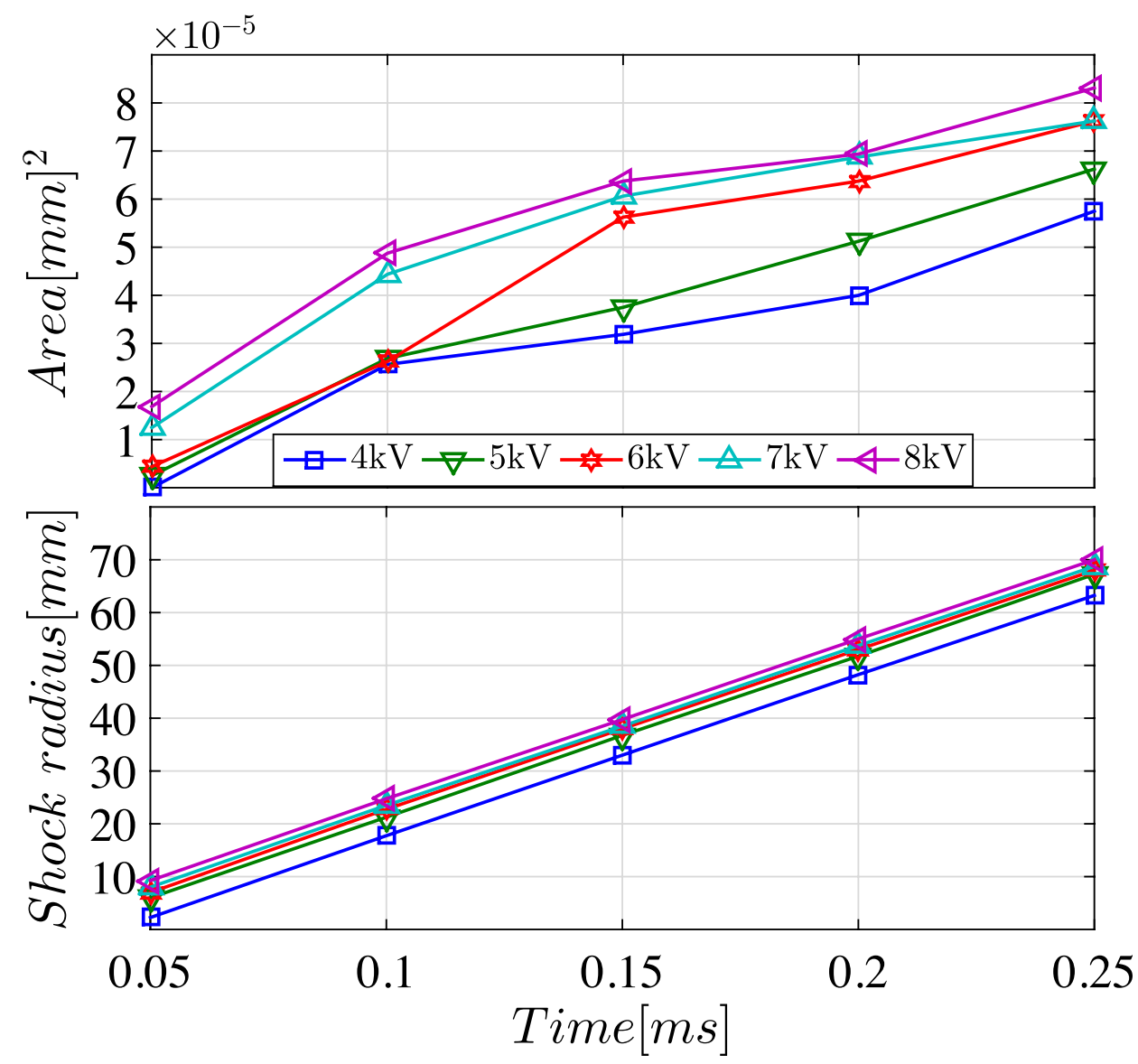

FIG. 15. Bright area method applied to single discharge case with different voltages (Top). Same cases processed with shockwave radius vs time ${ }^{11,12}$ (bottom). order to calibrate this method with respect to the shockwave radius vs time, as done in previous works. ${ }^{11,12}$

Phase locked Schlieren imaging acquisitions were carried out at $20 \mathrm{kHz}$ (i.e., $50 \mu$ s between consecutive frames) and exposure time was $2 \mu \mathrm{s}$. In Figure 15, results of the two methods are reported and compared. Discharge voltage was the only parameter and it ranged from 4 to $8 \mathrm{kV}$. It can be observed that the trends of the two methods are similar. Indeed, increasing voltage at a fixed instant time, a larger area suggests a higher energy deposited within the discharge volume. In the same way, for a fixed instant time, a larger shockwave radius indicates a more energetic discharge, i.e., a faster shock is generated during the first microseconds after a discharge. ${ }^{11}$ However, in order to see different velocities of shockwaves within the first microseconds after a discharge, an acquisition frequency of the order of $100 \mathrm{kHz}$ is required with a relatively high spatial resolution, which goes beyond our equipment capabilities.

\section{DISCUSSION}

An experimental investigation on the parameters affecting the energy deposition of a nanosecond dielectric barrier discharge plasma actuator was carried out. The experimental framework includes still air measurements by means of Schlieren technique, electrical characterization of materials, voltage measurements via back-current shunt technique in order to calculate the total energy input per pulse, and light intensity measurements of the pulsed discharge.
Results in still air show the formation of weak compression waves within the first microseconds after discharge, consistent with previous experimental observations. ${ }^{7,8,12}$ Additionally, a heated gas volume is observed in the vicinity of the actuator several milliseconds after the discharge. ${ }^{9,10,13}$ Expansion of the heated volume is acquired after each 10 discharged pulses. Estimation and comparison of these "hot areas" formed for different energy inputs (from 10 to 100 pulses) show an accumulation of deposited energy as each pulse is individually contributing to the growth of the estimated heated area. ${ }^{10,13}$

Results underlay a strong dependency of the energy deposited into the air within the discharge volume upon the thickness of the barrier as well as the material it is made out of. Moreover, a linear energy deposition relatively to the thickness of the barrier is observed regardless of the material of the barrier itself. Relative energy deposition rate is much lower for Silicone and PA and electrical measurements show higher efficiency of energy deposition for the case of Kapton. The size of the heated area in case of Kapton is one order of magnitude bigger than for the other two cases, for all the energy inputs. Light intensity acquisition has shown a more pronounced discharge for the case of dielectric barrier made out of PA. This phenomenon is due to the fact that the porosity of the material allows electron streamers to travel on preferential paths. Results have demonstrated qualitatively that efficiency of devices, such as plasma actuators, strongly depends upon the material used for the dielectric barrier and its own thickness. In general, these results 
demonstrate a higher efficiency achieved by a strong dielectric material concerning thermal energy deposition. It is concluded that a barrier of an ns-DBD plasma actuator should be as thin as possible in order to maximize the electrical field that produces plasma which ultimately delivers the thermal energy deposition required for any flow control task. However, size of the thickness affects the lifetime of the barrier itself. ${ }^{27,28}$ In order to minimize the thickness of a barrier, it is of primary importance to use a material with a very high dielectric strength. In order to maximize the extent of the plasma region, it would be preferred to have a material with a volumetric resistivity as small as possible. Nevertheless, the former and the latter material proprieties are competing with each other. Furthermore, for a barrier, it would be preferred to have a heat capacitance as small as possible and a thermal conductivity coefficient as small as possible in order to reduce at minimum the heat dissipated within the actuator itself.

\section{ACKNOWLEDGMENTS}

The authors would like to thank the $\mathrm{PhD}$ students Theodoros Michelis and Ilya Popov for the helpful discussion and PhD students Hamideh Khanbareh and Nan Zhong for their help with the material properties testing.

${ }^{1}$ E. Moreau, “Airflow control by non-thermal plasma actuators," J. Phys. D: Appl. Phys. 40, 605-636 (2007).

${ }^{2}$ T. C. Corke, M. L. Post, and D. M. Orlov, "Single dielectric barrier discharge plasma enhanced aerodynamics: Physics, modelling and applications," Exp. Fluids 46, 1-26 (2009).

${ }^{3}$ C. Enloe, T. McLaughlin, R. VanDyken, K. Kachner, E. Jumper, and T. Corke, "Mechanisms and responses of a single dielectric barrier plasma actuator: Plasma morphology," AIAA J. 42, 589-594 (2004).

${ }^{4}$ C. Enloe, T. McLaughlin, R. VanDyken, K. Kachner, E. Jumper, T. Corke, M. Post, and O. Haddad, "Mechanisms and responses of a single dielectric barrier plasma actuator: Geometric effects," AIAA J. 42, 595-604 (2004).

${ }^{5}$ C. Rethmel, J. Little, K. Takashima, A. Sinha, I. Adamovich, and M. Samimy, "Flow separation control using nanosecond pulse driven DBD plasma actuators," Int. J. Flow Control 3, 213-232 (2011).

${ }^{6}$ G. Correale, I. Popov, A. Rakitin, A. Starikovskii, S. Hulshoff, and L. Veldhuis, "Flow separation control on airfoil with pulsed nanosecond discharge actuator," in 49th AIAA Aerospace Sciences Meeting, Orlando, FL, 2011

${ }^{7}$ J. Little, K. Takashima, M. Nishihara, I. Adamovich, and M. Samimy, "Separation control with nanosecond-pulse-driven dielectric barrier discharge plasma actuators," AIAA J. 50, 350-365 (2012).

${ }^{8}$ D. V. Roupassov, A. A. Nikipelov, M. M. Nudnova, and A. Y. Starikovskii, "Flow separation control by plasma actuator with nanosecond pulsed-periodic discharge," AIAA J. 47, 168-185 (2009).

${ }^{9}$ T. Michelis, G. Correale, I. B. Popov, M. Kotsonis, D. Ragni, S. J. K. Hulshoff, and L. L. M. Veldhuis, "Disturbance introduced into a laminar boundary layer by a NS-DBD plasma actuator," in 51st AIAA Aerospace Science Meeting including the New Horizons Forum and Aerospace Exposition, 7-10 January 2013, Grapevine (Dallas/Ft. Worth Region), Texas.
${ }^{10}$ G. Correale, T. Michelis, D. Ragni, M. Kotsonis, and F. Scarano, "Nanosecond-pulsed plasma actuation in quiescent air and laminar boundary layer,” J. Phys. D: Appl. Phys. 47, 105201 (2014).

${ }^{11}$ K. Takashima, Y. Zuzeek, W. Lempert, and I. Adamovich, "Characterization of a surface dielectric barrier discharge plasma sustained by repetitive nanosecond pulses," Plasma Sources Sci. Technol. 20, 055009 (2011).

${ }^{12}$ N. Benard, N. Zouzou, A. Claverie, J. Sotton, and E. Moreau, "Optical visualization and electrical characterization of fast-rising pulsed dielectric barrier discharge for airflow control applications," J. Appl. Phys. 111, 033303 (2012).

${ }^{13}$ J. Zheng, Z. Zhao, J. Li, Y. Cui, and B. Khoo, "Numerical simulation of nanosecond pulsed dielectric barrier discharge actuator in a quiescent flow," Phys. Fluids 26, 036102 (2014).

${ }^{14}$ I. Popov and S. Hulshoff, Numerical Investigation of Instabilities in Free Shear Layer Produced by ns-DBD Actuator (World Academy of Science, Engineering and Technology, 2012), Vol. 6.

${ }^{15}$ F. Thomas, T. Corke, M. Iqbal, A. Kozlov, and D. Schatzman, "Optimization of dielectric barrier discharge plasma actuators for active aerodynamic flow control," AIAA J. 47, 2169-2178 (2009).

${ }^{16}$ T. Abe, Y. Takizawa, S. Sato, and N. Kimura, "A parametric experimental study for momentum transfer by plasma actuator," in 45th AIAA Aerospace Sciences Meeting, 8-11 January 2007, Reno, Nevada, 2007.

${ }^{17}$ D. Opaits, S. Zaidi, M. Shneider, and R. Miles, "Suppression of dielectric barrier discharge charge buildup using a partially conducting thin film," in 39th AIAA Fluid Dynamics Conference, 22-25 June 2009, San Antonio, Texas.

${ }^{18}$ A. Starikovskiy and R. Miles, "Dielectric barrier discharge control and flow acceleration enhancement by diode surface," in 51st AIAA Aerospace Sciences Meeting including the New Horizons Forum and Aerospace Exposition, 07-10 January 2013, Grapevine (Dallas/Ft. Worth Region), Texas.

${ }^{19}$ A. Starikovskii, A. Nikipelov, M. Nudnova, and D. Roupassov, "SDBD plasma actuator with nanosecond pulse-periodic discharge," Plasma Source Sci. Technol. 18, 034015 (2009)

${ }^{20}$ L. N. Dworsky, Modern Transmission Line Theory and Applications (Krieger Publishing Company, 1980).

${ }^{21}$ D. Opaits, M. Shneider, and R. Miles, "Electrodynamic effects in nanosecond-pulse-sustained long dielectric-barrier-discharge plasma actuators," Appl. Phys. Lett. 94, 061503 (2009).

${ }^{22}$ M. Kotsonis, "Diagnostics for characterization of plasma actuators," Meas. Sci. Technol. 26, 092001 (2015).

${ }^{23}$ T. Log and S. Gustafsson, Transient Plane Source (TPS) Technique for Measuring Thermal Transport Properties of Building Materials (John Wiley \& Sons Ltd, Chichester, UK, 1995), Vol. 19, pp. 43-49.

${ }^{24}$ U. Kogelschatz, B. Eliasson, and W. Egli, "Dielectric-barrier discharges. Principle and applications," J. Phys. IV 7(C4), C4-47-C4-66 (1997).

${ }^{25} \mathrm{R}$. Dawson and J. Little, "Characterization of nanosecond pulse driven dielectric barrier discharge plasma actuators for aerodynamic flow control," J. Appl. Phys. 113, 103302 (2013).

${ }^{26}$ A. Ndong, N. Zouzou, N. Benard, and E. Moreau, "Geometrical optimization of a surface DBD powered by a nanosecond pulsed high voltage," J. Electrostat. 71, 246-253 (2013).

${ }^{27}$ K. Kato, C. Breitsamter, and S. Obi, "Flow separation control over a Gö 387 airfoil by nanosecond pulse-periodic discharge," Exp. Fluids 55, 1795 (2014).

${ }^{28}$ A. Aba'A Ndong, N. Zouzou, N. Benard, and E. Moreau, "Effect of dielectric aging on the behavior of a surface nanosecond pulsed dielectric barrier discharge," IEEE Trans. Dielectr. Electr. Insul. 20, 1554-1560 (2013).

${ }^{29}$ K. Bayoda, N. Benard, and E. Moreau, "Elongating the area of plasma/ fluid interaction of surface nanosecond pulsed discharges," J. Electrostat. 74, 79-84 (2015). 